\title{
Topic: INCISIONAL HERNIA - Prophylactic Mesh
}

\author{
(C) Springer-Verlag 2014
}

\section{PO:102}

\section{SIMULTANEOUS REPAIR OF VENTRAL ABDOMI- NAL HERNIA AT THE TIME OF BARIATRIC SURGERY \\ A. Eng', W.H. Chan ${ }^{1}$, Y.C. Goh ${ }^{1}$, H.S. Ong ${ }^{1}$, W.K. Wong ${ }^{1}$ \\ ${ }^{\prime}$ Singapore General Hospital - Department of General Surgery, Singapore, SINGAPORE}

Introduction: Bariatric surgery is increasingly commonplace in Asia. The commonest procedure in Singapore is the sleeve gastrectomy. The incidence of ventral abdominal hernias such as incisional hernias is higher in the obese population. These patients often prefer to have their hernias fixed at the time of bariatric surgery rather than several months later after weight loss is achieved.

Methods: We review 3 patients who were scheduled for simultaneous laparoscopic sleeve gastrectomy and ventral hernia repair. The preoperative status as well as intraoperative details and postoperative outcome will be compared and we will review the literature on simultaneous bariatric surgery and hernia repair.

Results: 3 middle-aged female patients of BMI 39-44 were scheduled for laparoscopic sleeve gastrectomy and concurrent mesh repair of multiple ventral hernias. All 3 underwent preoperative CT scan to better assess the defects and sac contents. Two of the patients had had several abdominal operations before in the past and all three had symptoms of pain in the hernia. Just prior to surgery, one patient decided not to proceed with sleeve gastrectomy and she therefore had laparoscopic adhesiolysis and IPOM repair only. The two patients who had concurrent sleeve gastrectomy and hernia repair had excellent weight loss (EWL 70\% at one year) with no evidence of recurrence or infection. Of the two, one had mesh repair and the other only had primary closure of the extended midline fascial defect after extraction of the sleeved stomach and omentectomy specimen. The patient who elected to forego bariatric surgery gained an additional $6 \mathrm{~kg}$ and eventually had a recurrence of the hernia at 14 months after surgery.

Conclusion: Bariatric surgery at the time of repair of multiple ventral abdominal hernias is safe and in our experience likely to result in better satisfaction with good weight loss and no recurrence or infection. Patients who undergo only mesh repair of large incisional hernias without any weight loss procedure are more likely to have a recurrence and this is supported in the literature. The likelihood of infection of the mesh after gastrointestinal 'cutting' procedures is possible but has been reported to be less likely after sleeve gastrectomy than after gastric bypass. Given that our patients required significant laparoscopic adhesiolysis before the multiple hernias could be addressed, we believe that simultaneous sleeve gastrectomy and hernia repair is likely to be the preferred choice of initial procedure in these patients instead of a gastro-intestinal bypass procedure. If more weight loss is required later on, then a second-stage conversion to a bypass or BPD can be performed subsequently.

\section{PO:103}

\section{PARAOSTOMAL HERNIA PREVENTION -} SUGARBAKER TECHNIQUE

P. Cingolani' ${ }^{1}$ L D'Angelo, C Chwat, F Iudica, M Terrés, S Marchionatti, G Lemme

'Hospital Universitario Austral - Department of General Surgery, Pilar, ARGENTINA

Introduction: Paraostomal hernias present an incidence of $30-40 \%$, beeing clinically evidenced within the first 2 years after surgery. The use of a mesh reduces the incidence up to $4 \%$. Risk factors include obesity, malnutrition, immunosuppression, emergency surgery, infection, inflammatory or neoplasic diseases. Signs and symptoms include a local tumor, pain or intestinal obstruction.

Methods: Woman, 58 years old. Chief complaint: Anal epidermoid carcinoma. She completed Nigro scheme (radio and chemotherapy) Due to tumoral persistence a Miles Operation is decided. Colostomy is performed with Sugarbaker technique for paraostomal prevention.

Results: 1-Colon is exteriorized on the left lumbar region. 2-Colon is exteriorized on the left lumbar region. 3- Mesh fixation to anterior abdominal wall with transabdominal sutures and absorbable tackers. 4- Stoma fixation is completed

Conclusion: The high incidence of parastomal hernia and controversy and different results on its repair, make prevention a vital priority.

Mesh use for the reinforcement of abdominal wall during stoma creation, decreases the incidence of paraostomal hernias and prevents future reinterventions. 